\title{
The REASONS Survey: Resolved Millimeter Observations of a Large Debris Disk around the Nearby F Star HD 170773
}

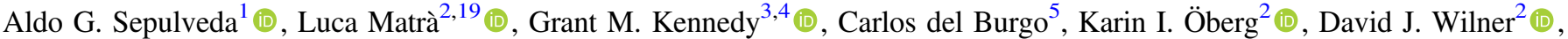 \\ Sebastián Marino $^{6}$ (D), Mark Booth ${ }^{7}$, John M. Carpenter $^{8}$ (D), Claire L. Davies ${ }^{9}$ (D), William R. F. Dent ${ }^{8,10}$, Steve Ertel ${ }^{10,11,12}$ (iD, \\ Jean-Francois Lestrade ${ }^{13}$, Jonathan P. Marshall ${ }^{14}$ (D), Julien Milli ${ }^{10}$ (D), Mark C. Wyatt ${ }^{15}$ (D), Meredith A. MacGregor ${ }^{16,20}$ (DD , and \\ Brenda C. Matthews ${ }^{17,18}$ (iD \\ ${ }^{1}$ Department of Physics \& Astronomy, The University of Texas at San Antonio 1 UTSA Circle San Antonio, TX 78249, USA; aldogsepulveda@gmail.com \\ ${ }^{2}$ Harvard-Smithsonian Center for Astrophysics 60 Garden Street Cambridge, MA 02138, USA \\ ${ }^{3}$ Department of Physics, University of Warwick, Gibbet Hill Road, Coventry, CV4 7AL, UK \\ ${ }^{4}$ Centre for Exoplanets and Habitability, University of Warwick, Gibbet Hill Road, Coventry, CV4 7AL, UK \\ ${ }^{5}$ Instituto Nacional de Astrofísica, Óptica y Electrónica, Luis Enrique Erro 1, Sta. Ma. Tonantzintla, 72840 Puebla, Mexico \\ ${ }^{6}$ Max Planck Institute for Astronomy, Königstuhl 17, D-69117 Heidelberg, Germany \\ ${ }^{7}$ Astrophysikalisches Institut und Universitätssternwarte, Friedrich-Schiller-Universität Jena, Schillergäßchen 2-3, D-07745 Jena, Germany \\ ${ }^{8}$ Joint ALMA Observatory (JAO), Avenida Alonso de Córdova 3107 Vitacura 7630355, Santiago, Chile \\ ${ }^{9}$ Astrophysics Group, School of Physics, University of Exeter, Exeter EX4 4QL, UK \\ ${ }^{10}$ European Southern Observatory, Avenida Alonso de Córdova 3107 Vitacura 7630355, Santiago, Chile \\ ${ }^{11}$ Large Binocular Telescope Observatory, 933 North Cherry Avenue, Tucson, AZ 85721, USA \\ 12 Steward Observatory, Department of Astronomy, University of Arizona, 993 N. Cherry Avenue, Tucson AZ 85721, USA \\ ${ }^{13}$ LERMA, Observatoire de Paris, PSL, CNRS, Sorbonne Universités, UPMC Univ., 61 avenue de l'Observatoire, Paris, France \\ ${ }^{14}$ Academia Sinica, Institute of Astronomy and Astrophysics, 11F Astronomy-Mathematics Building, NTU/AS campus, No. 1, Section 4, Roosevelt Road, Taipei \\ 10617, Taiwan \\ ${ }^{15}$ Institute of Astronomy, University of Cambridge, Madingley Road, Cambridge CB3 OHA, UK \\ ${ }^{16}$ Department of Terrestrial Magnetism, Carnegie Institution for Science, 5241 Broad Branch Road, Washington, DC 20015, USA \\ ${ }^{17}$ Department of Physics \& Astronomy, University of Victoria, 3800 Finnerty Road, Victoria, BC, V8P 5C2, Canada
${ }^{18}$ Herzberg Astronomy \& Astrophysics Programs, National Research Council of Canada, 5071 West Saanich Road, Victoria, BC, V9E 2E7, Canada \\ Received 2019 April 11; revised 2019 June 18; accepted 2019 June 19; published 2019 August 14
}

\begin{abstract}
Debris disks are extrasolar analogs to our own Kuiper Belt and they are detected around at least $17 \%$ of nearby Sun-like stars. The morphology and dynamics of a disk encode information about its history, as well as that of any exoplanets within the system. We used the Atacama Large Millimeter/submillimeter Array (ALMA) to obtain $1.3 \mathrm{~mm}$ observations of the debris disk around the nearby F5V star HD 170773. We image the face-on ring and determine its fundamental parameters by forward-modeling the interferometric visibilities through a Markov Chain Monte Carlo approach. Using a symmetric Gaussian surface density profile, we find a $71 \pm 4$ au wide belt with a radius of $193_{-3}^{+2}$ au, a relatively large radius compared with most other millimeter-resolved belts around late A/early F type stars. This makes HD 170773 part of a group of four disks around A and F stars with radii larger than expected from the recently reported planetesimal belt radius-stellar luminosity relation. Two of these systems are known to host directly imaged giant planets, which may point to a connection between large belts and the presence of long-period giant planets. We also set upper limits on the presence of $\mathrm{CO}$ and $\mathrm{CN}$ gas in the system, which imply that the exocomets that constitute this belt have $\mathrm{CO}$ and $\mathrm{HCN}$ ice mass fractions of $<77 \%$ and $<3 \%$, respectively. This is consistent with solar system comets and other exocometary belts.
\end{abstract}

Key words: circumstellar matter - planet-disk interactions - stars: individual (HD 170773) - techniques: interferometric

\section{Introduction}

Debris disks (also known as exocometary belts or planetesimal belts) are rings of dust, exocomets, and planetesimals analogous to our solar system's Kuiper Belt and are detected around at least $\sim 17 \%$ of Sun-like stars (Montesinos et al. 2016; Sibthorpe et al. 2018). Debris disks span different regions across planetary systems, including outer regions (colder belts typically located at tens of au from their host star) to inner regions (warmer belts at a few au that are generally more difficult to detect) (Kennedy \& Wyatt 2014; Ballering et al. 2017). These debris disks are intimately related to the formation of exoplanets and offer clues about the evolution and dynamical history of the system (Bowler 2016;

\footnotetext{
19 Submillimeter Array (SMA) Fellow.

${ }^{20}$ NSF Astronomy and Astrophysics Postdoctoral Fellow.
}

Wyatt 2018). Debris disks are generally thought to be maintained by a collisional cascade process, where destructive collisions between larger planetesimal bodies produce smaller planetesimals that further collide to produce the small dust grains that are observed (e.g., Wyatt et al. 2011; Hughes et al. 2018 and references therein).

The constituent dust grains reprocess starlight into radiation at longer wavelengths that are comparable to the sizes of the emitting bodies. The smallest dust grains are continuously blown out of the disk by the radiation pressure of the host star and additionally by stellar winds (Backman \& Paresce 1993). Thus, imaging debris disks in the infrared highlights emission (and scattering) from small grains that are blown out and may not delineate the true spatial architecture of the parent planetesimal belts. To probe the location of larger dust and planetesimals too massive to be strongly influenced by stellar 
winds and radiation pressure, observations must be made at longer wavelengths in the millimeter/submillimeter regime and must spatially resolve the disk (e.g., Augereau et al. 2001).

Understanding why debris disks form at their particular radii from their host stars is key to better understanding the physical mechanisms that create these disks. Matrà et al. (2018a) conducted a population study of $26 \mathrm{~mm}$ resolved debris disks and found a statistically significant correlation between the host-star luminosity and disk radius, which persists when accounting for potential observational biases. Constraining the radii for additional debris disks is the next step to further characterize this radius-luminosity relationship and to further explore other potential correlations. Empirically quantifying any correlations between disk parameters and host-star properties is an essential step to test and refine planet and debris disk formation models. The REsolved ALMA and SMA Observations of Nearby Stars (REASONS) survey, which is the follow up of the JCMT SCUBA-2 Observations of Nearby Stars (SONS) Legacy survey (Holland et al. 2017), aims to approximately double the sample size of millimeter-resolved debris disks.

We present new $1.3 \mathrm{~mm}$ observations of the debris disk around the nearby $(37.02 \pm 0.06$ pc: Gaia Collaboration et al. 2016, 2018; Bailer-Jones et al. 2018) F5V (Gray et al. 2006) star HD 170773. The infrared excess of HD 170773 was first detected by Sadakane \& Nishida (1986) with the Infrared Astronomical Satellite (IRAS), which is a general indication that a debris disk might be present in the system. Nilsson et al. (2010) first detected the disk at submillimeter wavelengths $(870 \mu \mathrm{m})$ using the APEX telescope and derived a disk radius of 170 au from the flux distribution. The disk was also detected and resolved at various far-IR wavelengths $(70,100,160 \mu \mathrm{m})$ by Moór et al. (2015) using the Herschel Space Observatory, with the weighted average disk radius reported to be $173.4 \pm 2.8$ au. Holland et al. (2017) then resolved the disk at $850 \mu \mathrm{m}$ using the JCMT and estimated the disk radius as $252 \pm 26$ au. We spatially resolve the debris disk around HD 170773 in the millimeter regime to probe the spatial properties of the parent belt that is traced by the millimeter dust grains too massive to be influenced by the stellar radiation and activity.

In Section 2 we describe our ALMA observations of HD 170773. Section 3 details our analysis of the observations and the process of modeling the disk and constraining the fundamental parameters in a Bayesian fashion using Markov Chain Monte Carlo (MCMC). Here we check the ALMA observations for $\mathrm{CO}$ and $\mathrm{CN}$ gas and set upper limits on the corresponding mass fractions of the exocomets. We also infer the stellar parameters of HD 170773 with a Bayesian method and using Gaia. We discuss the results in Section 4 and describe some of the consequences of our parameter constraints.

\section{ALMA Observations}

Three ALMA Band 6 observations at $1.3 \mathrm{~mm}$ (211-275 GHz) of HD 170773 were made on 2018 April 29, May 3, and June 5. The phase center of the observations is at the proper motion-corrected J2000 stellar position. The interferometric visibility data was fully calibrated by the ALMA observatory using their pipeline. Table 1 summarizes the observing parameters. The ALMA interferometer samples the Fourier transform of the sky brightness distribution, resulting in complex valued visibilities in $u-v$ space. Observing HD 170773 with both the Atacama Compact Array (ACA) and the $12 \mathrm{~m}$ array provides greater coverage of the $u-v$ visibility space that in turn better recovers extended emission in the disk image.

The correlator setup included four $2 \mathrm{GHz}$ wide spectral windows, two of which were centered at 243.1 and $245.1 \mathrm{GHz}$ at low spectral resolution for continuum, although all four were used in obtaining the HD 170773 continuum image. To obtain the dust continuum image from the visibilities in $u-v$ space, we first average the observations in frequency to reduce the data size, then concatenate all three observations. We then use the tclean task of CASA 5.4.1 (McMullin et al. 2007) to inverse transform and deconvolve the visibility data into the $1.3 \mathrm{~mm}$ CLEAN continuum emission image. We apply a Gaussian $u-v$ taper of $2^{\prime \prime}$ and a Briggs weighting factor of 0.5 to the visibilities in the imaging process. The Gaussian $u-v$ taper boosts the signal-to-noise ratio $(\mathrm{S} / \mathrm{N})$ of the ring at the cost of resolution.

The other two spectral windows were centered at 230.1 and 227.2 GHz at high spectral resolution to cover the $\mathrm{CO} J=2-1$ transition (at $230.538 \mathrm{GHz}$ ) and the $\mathrm{CN} N=2-1$ transitions (where we focus on the strongest fine and hyperfine structure transition, at $226.875 \mathrm{GHz}$ ). The spectral resolution of these spectral windows is $\sim 1.28 \mathrm{~km} \mathrm{~s}^{-1}$ (for a channel width of $488.281 \mathrm{kHz}$ or $0.64 \mathrm{~km} \mathrm{~s}^{-1}$ ). We first subtracted continuum emission from the visibilities using the CASA uvcontsub task, then proceeded to imaging (with the same weighting and $u-v$ taper as the continuum data set) to produce $\mathrm{CO}$ and $\mathrm{CN}$ data cubes covering $\pm 50 \mathrm{~km} \mathrm{~s}^{-1}$ from the expected stellar radial velocity $\left(-16 \pm 1 \mathrm{~km} \mathrm{~s}^{-1}\right.$ in the heliocentric frame; Gaia Collaboration et al. 2018).

\section{Analysis}

\subsection{3 mm Dust Continuum Image}

Figure 1 shows the $1.3 \mathrm{~mm}$ continuum emission of HD 170773. The noise level, measured as the rms in a region free from emission, is $\sigma_{\mathrm{rms}}=2.8 \times 10^{-2} \mathrm{mJy} \mathrm{beam}^{-1}$ where the beam size is 2 !' 15 by 1 !' 90 . The peak signal is $3.8 \times 10^{-1} \mathrm{mJy}$ beam $^{-1}$, yielding a peak $\mathrm{S} / \mathrm{N}$ of 14 . The disk is close to face-on and the distance from the host star to the radial emission peak along the disk major axis is about $\sim 5^{\prime \prime}$ ( $\sim 185 \mathrm{au}$ ). Although we use a $u-v$ taper to boost the $\mathrm{S} / \mathrm{N}$ of the image (Section 2), the width is clearly resolved when imaging with no $u-v$ taper. The continuum image shows a tentative asymmetry where the SE disk emission may be more pronounced. However, the measured peak signal in the SE quadrant is only $\sim 2 \sigma_{\text {rms }}$ higher than the peak signal measured in the NW quadrant, which means there is not significant evidence for an asymmetry. We also compared the flux density of the NW and SE halves of the disk and found no significant difference.

The $1.3 \mathrm{~mm}$ flux density of the disk was estimated in several ways. The flux density of the disk measured in the $>3 \sigma_{\text {rms }}$ region of the ring is $5.04 \pm 0.52 \mathrm{mJy}$ including an absolute flux uncertainty of $10 \%$ added in quadrature. Similarly, the flux density is $5.22 \pm 0.54 \mathrm{mJy}$ when the flux interior to the ring is included in the measurement. The flux density of the $>2 \sigma_{\text {rms }}$ region of the ring together with the interior region is $5.38 \pm 0.56 \mathrm{mJy}$. This is less than the flux density obtained from visibility modeling $(6.2 \pm 0.2 \mathrm{mJy}$, Section 3.3). The latter extrapolates to baselines shorter than probed by the ACA based on an assumed disk structure to recover emission missing 
Table 1

Summary of ALMA Observation Parameters

\begin{tabular}{|c|c|c|c|}
\hline Parameter & 2018 Apr 29 & 2018 May 3 & 2018 Jun 5 \\
\hline No. antennas & 10 & 12 & 50 \\
\hline Antenna size & $7 \mathrm{~m}$ & $7 \mathrm{~m}$ & $12 \mathrm{~m}$ \\
\hline Time on target & $34 \mathrm{~m} 49 \mathrm{~s}$ & $34 \mathrm{~m} 49 \mathrm{~s}$ & $14 \mathrm{~m} 12 \mathrm{~s}$ \\
\hline J2000 pointing R.A. & $18 \mathrm{~h} 33 \mathrm{~m} 01.056 \mathrm{~s}$ & $18 \mathrm{~h} 33 \mathrm{~m} 01.056 \mathrm{~s}$ & $18 \mathrm{~h} 33 \mathrm{~m} 01.057 \mathrm{~s}$ \\
\hline J2000 pointing decl. & $-39^{\circ} 53^{\prime} 32^{\prime \prime} 744$ & $-39^{\circ} 53^{\prime} 32^{\prime \prime} 745$ & $-39^{\circ} 53^{\prime} 32^{\prime \prime} 752$ \\
\hline Min/max baseline & $8.9-48.9 \mathrm{~m}$ & $8.9-48.9 \mathrm{~m}$ & $15.0-360.6 \mathrm{~m}$ \\
\hline $\operatorname{Min} / \max \mathrm{PWV}$ & $1.30-1.87 \mathrm{~mm}$ & $0.42-0.63 \mathrm{~mm}$ & $1.38-1.50 \mathrm{~mm}$ \\
\hline Gain calibrator & $\mathrm{J} 1802-3940$ & $\mathrm{~J} 1802-3940$ & $\mathrm{~J} 1802-3940$ \\
\hline Passband/flux calibrator & J1924-2914 & J1924-2914 & J1924-2914 \\
\hline Primary beam FWHM & $46 " .0$ & $46 " .0$ & $26 " .9$ \\
\hline
\end{tabular}

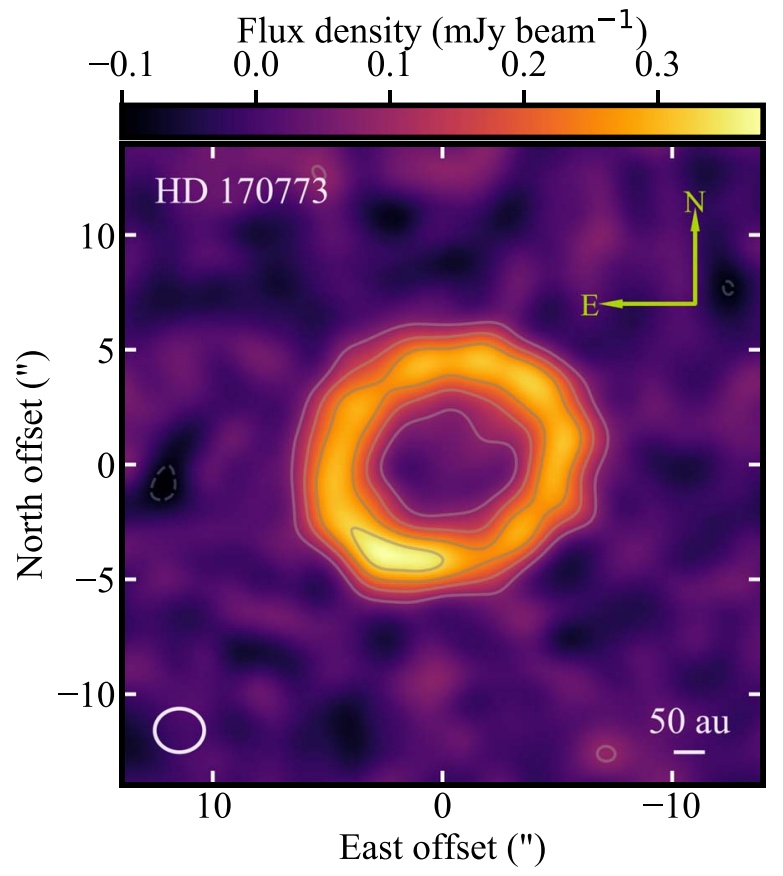

Figure 1. Combined ACA + $12 \mathrm{~m}$ array CLEAN image of HD 170773 dust continuum emission at $1.3 \mathrm{~mm}$ where a Gaussian $u-v$ taper of $2^{\prime \prime}$ and a Briggs weighting factor of 0.5 are used. Contours are drawn at the levels: $[-3,3,6,9,12] \times\left(\sigma_{\mathrm{rms}}=2.8 \times 10^{-2} \mathrm{mJy} \mathrm{beam}^{-1}\right)$. The beam size is indicated in the lower left corner of the image and measures 2 ". 15 by 1 !"90 with a position angle of $89^{\circ} .9$ (east of north).

from the image. This measurement $(6.2 \pm 0.6 \mathrm{mJy}$ when including the absolute flux uncertainty) is shown, along with other photometry (see Table 2 for the complete list) and Spitzer IRS spectroscopy of the HD 170773 system (Lebouteiller et al. 2011), in Figure 2.

We fit grids of star and disk models to the data, using synthetic photometry of the models to fit photometry, and resampled model spectra to fit the IRS spectrum. The model parameters are the stellar temperature (fit with a PHOENIX model atmosphere, Husser et al. 2013) and normalization (i.e., solid angle), and the disk temperature, normalization, and two "modified blackbody" parameters (the Planck function is divided by $\lambda^{\beta}$ beyond $\lambda_{0} \mu \mathrm{m}$ as a simple means to model the inefficient grain emission at long wavelengths). The best-fitting model parameters are found with the MultiNest code (Feroz et al. 2009), with both the stellar and disk parameters found simultaneously.
Table 2

Photometry Used to Generate Figure 2

\begin{tabular}{|c|c|c|c|c|}
\hline $\begin{array}{l}\text { Instrument/ } \\
\text { Filter }\end{array}$ & $\begin{array}{c}\text { Waveband } \\
(\mu \mathrm{m})\end{array}$ & Photometry & Unit & Reference \\
\hline$\overline{U-B^{\mathrm{a}}}$ & $\ldots$ & $-0.06 \pm 0.03$ & mag & 1 \\
\hline Strömgren $c_{1}{ }^{\mathrm{a}}$ & $\ldots$ & $0.48 \pm 0.02$ & mag & 2 \\
\hline$B_{T}$ & 0.42 & $6.71 \pm 0.02$ & mag & 3 \\
\hline Strömgren $m_{1}{ }^{\mathrm{a}}$ & $\ldots$ & $0.15 \pm 0.01$ & mag & 2 \\
\hline$B-V^{\mathrm{a}}$ & $\ldots$ & $0.42 \pm 0.02$ & mag & 1 \\
\hline$b-y^{\mathrm{a}}$ & $\ldots$ & $0.28 \pm 0.01$ & mag & 2 \\
\hline$V_{T}$ & 0.53 & $6.27 \pm 0.01$ & mag & 3 \\
\hline$H_{P}$ & 0.54 & $6.32 \pm 0.01$ & mag & 4 \\
\hline$V$ & 0.55 & $6.23 \pm 0.02$ & mag & 1 \\
\hline$J$ & 1.2 & $5.42 \pm 0.03$ & mag & 5 \\
\hline$H$ & 1.6 & $5.28 \pm 0.04$ & mag & 5 \\
\hline$K_{S}$ & 2.2 & $5.20 \pm 0.02$ & mag & 5 \\
\hline WISE W1 & 3.4 & $5.21 \pm 0.14$ & mag & 6 \\
\hline WISE W2 & 4.6 & $5.05 \pm 0.06$ & mag & 6 \\
\hline$A K A R I / I R C$ & 9.0 & $490 \pm 14$ & mJy & 7 \\
\hline WISE W3 & 12 & $5.22 \pm 0.05$ & mag & 6 \\
\hline WISE W4 & 22 & $5.08 \pm 0.07$ & mag & 6 \\
\hline Spitzer/MIPS & 24 & $67 \pm 1$ & mJy & 8 \\
\hline Herschel/PACS & 70 & $794 \pm 24$ & mJy & $9^{b}$ \\
\hline Spitzer/MIPS & 70 & $788 \pm 79$ & mJy & 10 \\
\hline Herschel/PACS & 100 & $1071 \pm 67$ & mJy & $9^{b}$ \\
\hline Herschel/PACS & 160 & $863 \pm 44$ & mJy & $9^{\mathrm{b}}$ \\
\hline Herschel/SPIRE & 250 & $358 \pm 26$ & mJy & 11 \\
\hline Herschel/SPIRE & 350 & $177 \pm 16$ & mJy & 11 \\
\hline JCMT/SCUBA-2 & 450 & $<135$ & mJy & 12 \\
\hline Herschel/SPIRE & 500 & $67 \pm 10$ & $\mathrm{mJy}$ & 11 \\
\hline JCMT/SCUBA-2 & 850 & $26 \pm 2$ & $\mathrm{mJy}$ & 12 \\
\hline APEX/LABOCA & 870 & $18 \pm 5$ & mJy & 13 \\
\hline ALMA & 1300 & $6.2 \pm 0.6$ & $\mathrm{mJy}$ & 9 \\
\hline
\end{tabular}

Notes.

a Colors (i.e., flux ratios) are fit directly.

b PACS fluxes were derived with apertures as described in Sibthorpe et al. (2018).

References. (1) Mermilliod (2006), (2) Paunzen (2015), (3) Høg et al. (2000), (4) ESA (1997), (5) Cutri et al. (2003), (6) Wright et al. (2010), (7) Ishihara et al. (2010), (8) IRSA, https://irsa.ipac.caltech.edu, (9) This work, (10) Chen et al. (2014a), (11) Schulz et al. (2017), (12) Holland et al. (2017), (13) Nilsson et al. (2010).

We find best-ft values of $T_{\text {eff }}=6640 \mathrm{~K}$ for the stellar temperature, and $40 \mathrm{~K}$ for the "modified" blackbody (the blackbody function is divided by $\lambda^{0.9}$ beyond $200 \mu \mathrm{m}$ ) of the disk. The $850 \mu \mathrm{m}$ flux from JCMT observations is higher than, but still within $\sim 3 \sigma$ of the best-fit model. The overall 


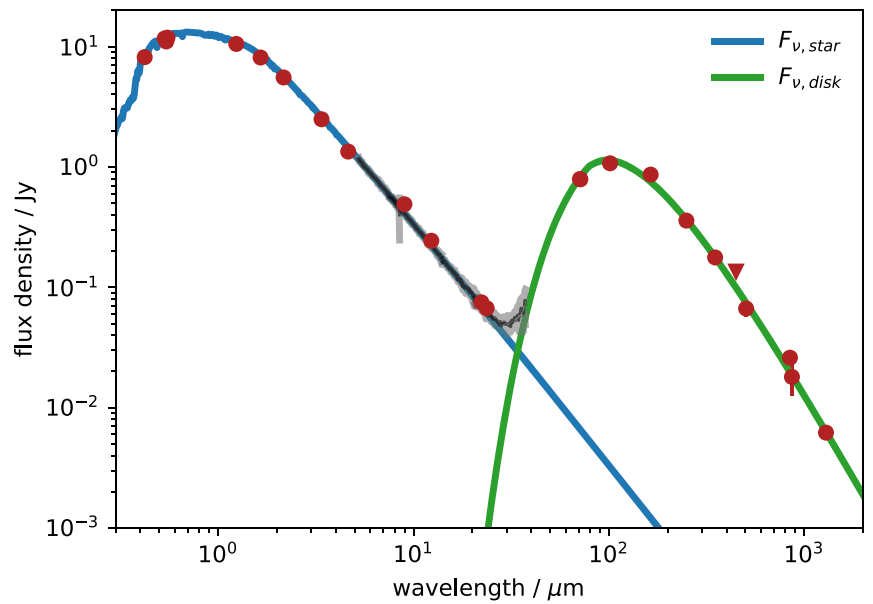

Figure 2. Flux distribution for HD 170773. Dots show photometry and the black and gray lines show the Spitzer IRS spectroscopy and uncertainty. The downward-pointing triangle shows the JCMT $450 \mu \mathrm{m}$ non-detection upper limit. The blue line shows the best-fit stellar photosphere, and the green line the best-fit modified blackbody for the disk.

millimeter spectral slope beyond $200 \mu \mathrm{m}$ is $\alpha_{\mathrm{mm}}=2.87 \pm$ 0.04 . This is comparable to the millimeter spectral slopes for a sample of 15 other disks (MacGregor et al. 2016). Here the fractional luminosity of the disk $\left(L_{\text {disk }} / L_{\star}\right)$ is $f=(5.0 \pm 0.1) \times$ $10^{-4}$. We note a $3.6 \sigma$ flux excess at $24 \mu \mathrm{m}$ that this model does not account for. One possible explanation could be the presence of a warm inner disk which is not detected in our ALMA observations.

\subsection{Disk Modeling and Fitting}

To precisely constrain the fundamental spatial properties of the debris disk, we forward-model the disk surface density profile using an azimuthally symmetric vertically thin Gaussian ring, which is a commonly used model for describing millimeterresolved debris disks (e.g., MacGregor et al. 2015; Booth et al. 2017; Marino et al. 2017; Su et al. 2017; Marshall et al. 2018; Matrà et al. 2019b). The mean $(\mu)$ of the Gaussian represents the radius of the disk at peak surface density, and the standard deviation $(\sigma)$ represents the spread of the surface density with respect to the peak. The temperature dependence as a function of radius (assuming blackbody dust grains) is also factored into our surface brightness profile as $S_{B} \propto B_{\nu}(T) \propto T(r) \propto \frac{1}{\sqrt{r}}$, where $B_{\nu}(T)$ is the Planck function which, for long wavelengths, is proportional to the inverse square root of the radius. The complete radial dependence of surface brightness in our model is given by

$$
S_{B}\left(f_{0}, \sigma, \mu ; r\right) \propto \frac{f_{0}}{\sqrt{r}} e^{-(r-\mu)^{2} / 2 \sigma^{2}} .
$$

This model is parameterized by $\sigma, \mu$, and $f_{0}$, where $f_{0}$ represents the integrated flux of the belt. In addition to these model parameters, we also account for four additional parameters which describe the line-of-sight inclination $(i)$, position angle of the major axis measured east of north $(P A)$, and offsets of the belt's geometric center from the phase center of the observation ( $\Delta R . A$. and $\Delta d e c l$.). We have accounted for potential systematic errors in the values of the visibility weights delivered by ALMA, which have been found in other data sets (e.g., Kennedy et al. 2018; Marino et al. 2018), and which could also otherwise mean that our uncertainties are underestimated. This is done by including a free parameter multiplied by the weights of each of the three observational data sets.

The galario (Tazzari et al. 2018) package is utilized to Fourier transform the model image at the $u-v$ locations of our ALMA data to calculate the $\chi^{2}$ of the model given the data. To derive the best-fitting synthetic model visibility data set, the posterior probability distributions of our model parameters are explored using the emcee (Foreman-Mackey et al. 2013) package, the affine-invariant ensemble sampler implementation of MCMC (Goodman \& Weare 2010) in Python. We use a likelihood function proportional to $\exp \left(-\chi^{2} / 2\right)$ and use linearly uniform priors on all our model parameters $f_{0}, \sigma, \mu$, $i, P A, \Delta R . A$., and $\Delta d e c l$. We initialize an ensemble of 1024 walkers each sampling from the parameter space for $1.25 \times 10^{4}$ time steps with a burn-in strip size of $2.5 \times 10^{3}$. We assessed the convergence of the Markov chains by comparing their length to their integrated autocorrelation times for each parameter (estimated with the emcee package). We find that all the chains are at least $\sim 67$ times their integrated autocorrelation time, ensuring convergence. The chains were also visually inspected to confirm that a steady state was reached.

\subsection{Model-fitting Results}

Figure 3 shows a corner plot summary of the emcee results. We summarize the best-fit values of the disk parameters (taken as the median value for each respective posterior distribution) together with their $68 \%$ confidence intervals in Table 3. Our constraint for $\mu$ yields a radius at peak emission constraint of $193_{-3}^{+2}$ au. The difference of this measurement compared with the constraints of Moór et al. (2015) and Holland et al. (2017) is likely due to differences in modeling formalism (e.g., Moór et al. 2015 use an annulus of constant surface brightness), although the radii are still broadly consistent. The inclination and position angle of $33_{-2}^{\circ+1}$ and $114_{-3}^{\circ+2}$ are consistent with the constraints of Moór et al. (2015), who reported the parameters as $31^{\circ} .3 \pm 1.7$ and $118^{\circ} .3 \pm 3.2$, respectively. The phase center offsets ( $\Delta R . A$. and $\Delta d e c l$.) are consistent with zero, indicating the disk's geometrical center is consistent with the stellar location, in contrast with some other debris disks found to be eccentric (e.g., Fomalhaut, Kalas et al. 2005; MacGregor et al. 2017). The width of the disk, interpreted as the FWHM of the Gaussian surface density distribution, is $71 \pm 4$ au. This makes the disk somewhat narrow $(\Delta R / R=0.37)$, which is common given the current observations of debris disks (Hughes et al. 2018).

In Figure 4 we show the image of the synthetic best-fit model of the disk along with the corresponding image of the residual visibilities after subtraction of this best-fit model. The residual image is consistent with noise and no significant emission at the $>4 \sigma_{\text {rms }}$ level is present at the disk location, indicating that the best-fit model parameters are consistent with the data and also confirming the lack of significant evidence for the possible asymmetry noted in Section 3.1. We only note a marginal positive $3 \sigma_{\text {rms }}$ residual to the NW and slightly offset from the stellar location. We also show the deprojected visibility plot of the data and best-fit model as Figure 5. The imaginary visibilities being consistent with zero further supports the axisymmetric model.

The asymmetric shape of the radius (as well as $i$ and $P A$ ) posterior distributions in Figure 3, in addition to a potential 


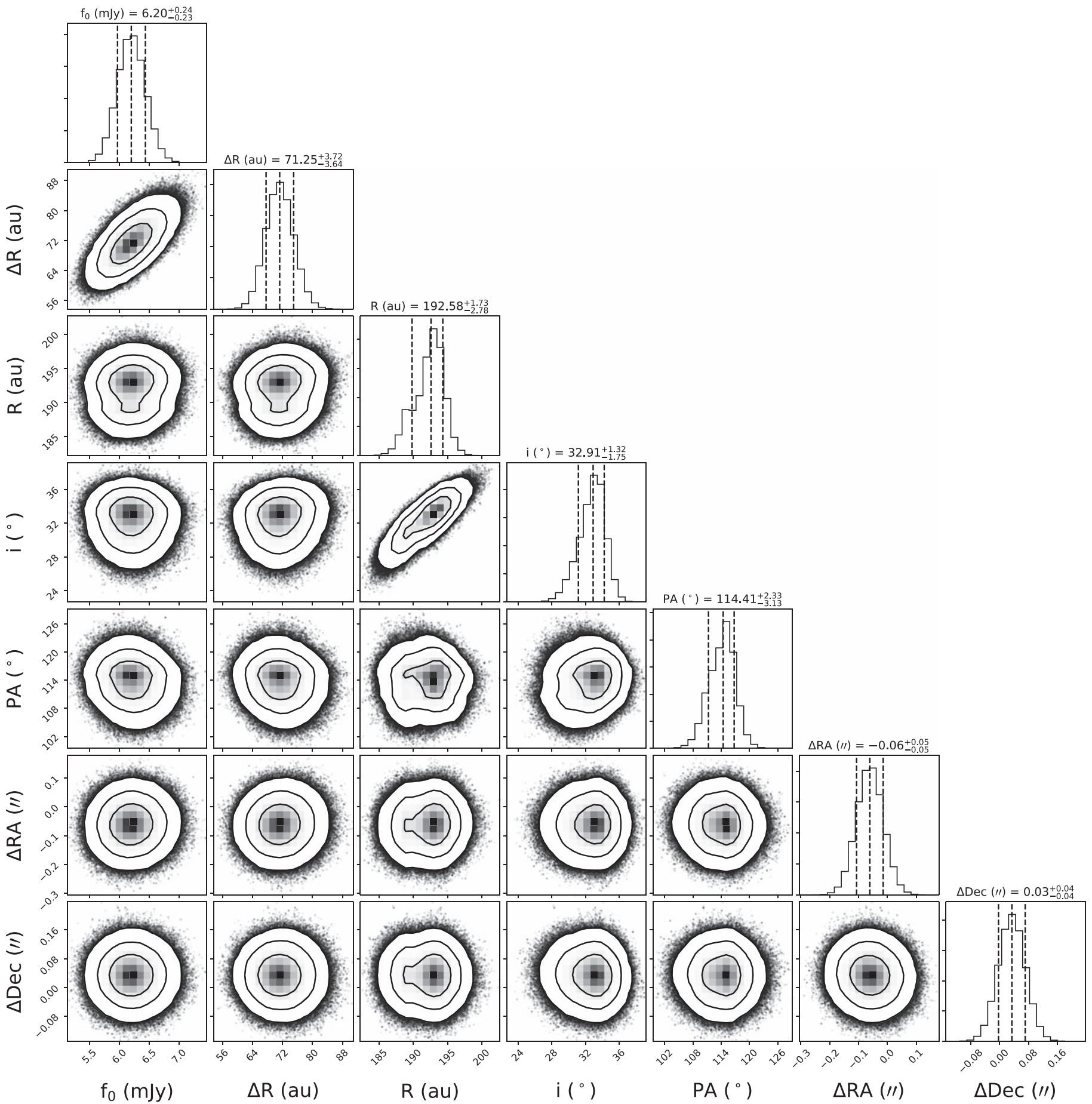

Figure 3. Corner plot summary of the emcee run results showing the marginalized posterior probabilities for the disk parameters (1D histograms) as well as the 2D projections for each combination of the disk parameters (2D histograms). The 1D posterior probabilities have their $16 \%, 50 \%$, and $84 \%$ quantiles displayed as vertical dashed lines and with their values listed above each respective distribution. The contours of the 2D histograms are displayed for $68 \%, 95 \%$, and $99.7 \%$ density levels.

marginal over subtraction of the ring in Figure 4 (right panel), suggests a potential skewness of the Gaussian distribution we used as our parametric model for the surface density. We addressed this by remodeling the disk with more freedom for the standard deviation of the Gaussian profile to differ in the inward and outward radial directions. Here, $\sigma$ is replaced by two free parameters where now $\sigma_{\text {in }}$ is defined for $r<r_{0}$ and $\sigma_{\text {out }}$ for $r>r_{0}$. This second model is otherwise identical to the first. While visibility fitting with this asymmetric surface density model resulted in a marginally better $\chi^{2}$ value, we found that due to the additional free parameter it did not describe the data significantly better than the symmetric density model. This was assessed using the Bayesian Information Criterion (BIC; Schwarz 1978), where we find a $\triangle \mathrm{BIC}$ value of 7.3 favoring the symmetric density model.

\subsection{Constraints on $\mathrm{CO}$ and $\mathrm{CN}$ Line Emission}

No clear detection is seen in the data cubes around the frequency of the $\mathrm{CO} J=2-1$ line and the $\mathrm{CN} N=2-1$, 
Table 3

Fundamental Disk Parameter Constraints

\begin{tabular}{|c|c|c|c|}
\hline Parameter & Short Description & Best Fit & $\begin{array}{l}\text { 68\% Confidence } \\
\text { Interval }\end{array}$ \\
\hline$f_{0}(\mathrm{mJy})$ & Disk flux & 6.2 & $(+0.2,-0.2)$ \\
\hline$\sigma\left({ }^{\prime \prime}\right)$ & $\begin{array}{l}\text { Standard deviation of radial } \\
\text { surface density distribution }\end{array}$ & 0.82 & $(+0.04,-0.04)$ \\
\hline$\mu\left({ }^{\prime \prime}\right)$ & $\begin{array}{l}\text { Disk radius at peak surface } \\
\text { density }\end{array}$ & 5.20 & $(+0.05,-0.08)$ \\
\hline$i\left(^{\circ}\right)$ & Line-of-sight inclination & 33 & $(+1,-2)$ \\
\hline$P A\left({ }^{\circ}\right)$ & Position angle (east of north) & 114 & $(+2,-3)$ \\
\hline$\left.\Delta R . A .{ }^{(\prime)}\right)$ & R.A. offset & -0.06 & $(+0.05,-0.05)$ \\
\hline$\Delta \operatorname{Decl} .\left({ }^{\prime \prime}\right)$ & decl. offset & 0.03 & $(+0.04,-0.04)$ \\
\hline$R(\mathrm{au})$ & $\begin{array}{l}\text { Disk radius at peak surface } \\
\text { density }\end{array}$ & 193 & $(+2,-3)$ \\
\hline$\Delta R(\mathrm{au})$ & Width of disk & 71 & $(+4,-4)$ \\
\hline
\end{tabular}

Note. We report the best-fit values as the median value of the resulting posterior distribution, and the $68 \%$ interval as the confidence interval. The parameters directly involved in the emcee runs lie above the solid line. Below the solid line are the parameters derived from the emcee results, where the radius $(R)$ is $\mu$ converted to au and the disk width $\Delta R$ is the FWHM $(2 \sqrt{2 \ln 2} \sigma)$ converted to au.

$J=5 / 2-3 / 2$ transition, where the latter is composed of three, blended, hyperfine components $(F=7 / 2-5 / 2,5 / 2-3 / 2$ and $3 / 2-1 / 2$ ). Given that the disk is resolved over many spatial, and potentially spectral, resolution elements, we employ the spectro-spatial filtering technique of Matrà et al. (2015, 2017) to boost the $\mathrm{S} / \mathrm{N}$ by assuming $\mathrm{CO}$ and $\mathrm{CN}$ are co-located with the dust and in Keplerian velocity (where both rotation directions were tested) around the star (of mass $1.29 M_{\odot}$, Section 3.5). This is what is expected for gas released from exocometary ices within the collisional cascade that also produces the dust, as observed in several other systems (e.g., Marino et al. 2016; Matrà et al. 2017).

No detection is achieved; we set an upper limit $(3 \sigma)$ of 35 and $47 \mathrm{mJy} \mathrm{km} \mathrm{s}^{-1}$ on the integrated line flux of the $\mathrm{CO}$ and $\mathrm{CN}$ transitions, respectively. This was calculated from the rms of the spectro-spatially filtered spectra, multiplied by the effective bandwidth of the instrument (2.667 times the channel width) assuming, as expected, that the spectro-spatially filtered line is close to unresolved spectrally. This uncertainty was added in quadrature to a $10 \%$ absolute flux uncertainty expected from ALMA observations.

We then derived $\mathrm{CO}$ and $\mathrm{CN}$ gas mass upper limits from the observed fluxes, using the nonlocal thermodynamic equilibrium (NLTE) excitation code of Matrà et al. $(2015,2018 \mathrm{~b})$ in the optically thin assumption. We explore the full range of collider densities between the regime where excitation is dominated by collisions (LTE) and radiative absorption/ emission, and temperatures between 10 and $250 \mathrm{~K}$. This allows us to derive upper limits of $1-14 \times 10^{-6}$ and $1.3-8.0 \times$ $10^{-8} M_{\oplus}$ on the $\mathrm{CO}$ and $\mathrm{CN}$ gas masses, respectively.

We then assume that any gas that may be present is being released from exocometary ice through a steady-state collisional cascade and destroyed through photodissociation at the same rate as it is produced. Photodissociation at the $193 \mathrm{au}$ radius of the belt around an F star such as HD 170773 is dominated by the interstellar radiation field, leading to photodissociation timescales of $\sim 120$ and $\sim 61 \mathrm{yr}$ for $\mathrm{CO}$ and CN (Heays et al. 2017). Given that $\mathrm{HCN}$ is the main parent molecule producing $\mathrm{CN}$ via photodissociation, we can use $\mathrm{CN}$ to probe the exocometary $\mathrm{HCN}$ ice content (Matrà et al. 2018b).

As long as all $\mathrm{CO}$ and $\mathrm{HCN}$ are released from solids by the time these are ground down to the smallest size in the collisional cascade, the $\mathrm{CO}$ and/or $\mathrm{HCN}$ release is proportional to the mass-loss rate of the belt (see Section 4.1 in this work and Equation (2) in Matrà et al. 2017), and can be used to extract the ice mass fraction in exocomets. Around HD 170773, we estimate an upper limit to the $\mathrm{CO}$ and $\mathrm{HCN}$ exocometary mass fraction of $<77 \%$ and $<3 \%$, respectively. The $\mathrm{CO}$ limit is consistent with $\mathrm{CO}$ mass fractions of a few to a few tens of percent derived from detection around other exocometary belts, as well as solar system comets (e.g., Mumma \& Charnley 2011) assuming a rock/ice ratio of $\sim 4$ as measured in comet $67 \mathrm{P}$ (Rotundi et al. 2015).

\subsection{Stellar Parameters}

The stellar parameters of HD 170773, i.e., age, mass, luminosity, effective temperature, surface gravity, and radius, were inferred by employing the absolute $G$ magnitude $(6.1040 \pm 0.0004 \mathrm{mag}$, obtained from the apparent $\mathrm{G}$ magnitude and the parallax) and BP-RP color from Gaia DR2 (0.5691 $\pm 0.0059 \mathrm{mag}$, Gaia Collaboration et al. 2016, 2018; Lindegren et al. 2016), as well as $[\mathrm{Fe} / \mathrm{H}]$ (assumed to be solar, $0.00 \pm 0.20 \mathrm{dex})$, all three as input parameters using the Bayesian approach applied in del Burgo \& Allende Prieto (2016, 2018). We inferred that HD 170773 is most likely a main-sequence star, and show the derived parameters in Table 4.

To infer the stellar parameters, we downloaded and arranged a grid of PARSEC isochrones (version 1.2S, Bressan et al. 2012; Chen et al. 2014b, 2015; Tang et al. 2014), using the synthetic photometry from Evans et al. (2018). The iron-tohydrogen ratio $[\mathrm{Fe} / \mathrm{H}]$ ranges from -2.18 to 0.50 , in steps of $0.02 \mathrm{dex}$, the age goes from $200 \mathrm{Myr}$ to $13.5 \mathrm{Gyr}$, in steps of $5 \%$, and the initial mass ranges from $0.09 M_{\odot}$ to the highest mass established by the stellar lifetimes, in irregular steps that properly sample the slow and fast evolutionary phases. The absolute maxima for the initial mass and actual mass in the grid are $350.0 M_{\odot}$ and $345.2 M_{\odot}$, respectively. For a more detailed description, see del Burgo \& Allende Prieto (2018).

\section{Discussion}

\subsection{Collisional Cascade Status}

The blowout of the smallest dust grains in the debris disk due to radiation pressure, which are themselves created by the destruction of larger bodies, results in mass loss over time. Consequently, older debris disks will be less luminous and are harder to detect than younger disks. Matrà et al. (2017) derive a simple equation for the mass-loss rate of these smallest grains assuming a steady-state collisional cascade model, given as $\dot{M}_{D_{\min }}=1.2 \times 10^{3} R^{1.5} \Delta R^{-1} f^{2} L_{\star} M_{\star}{ }^{-0.5}$ where $R$ is in au, $L_{\star}$ is in $L_{\odot}$, and $M_{\star}$ is in $M_{\odot}$. Adopting the best-fit values of these parameters from Section 3 yields a mass-loss rate of $\dot{M}_{D_{\min }}=$ $3.6 \times 10^{-2} M_{\oplus} \mathrm{Myr}^{-1}$ for HD 170773. Assuming that mass loss has been ongoing at this constant rate for the age of the star, the total mass lost for HD 170773 is $54 M_{\oplus}$ when using an age of $1.5 \mathrm{Gyr}$ (Section 3.5). We note that there is substantial uncertainty in the age of HD 170773, as pinning down precise stellar ages is generally difficult. If the age of HD 170773 is as 


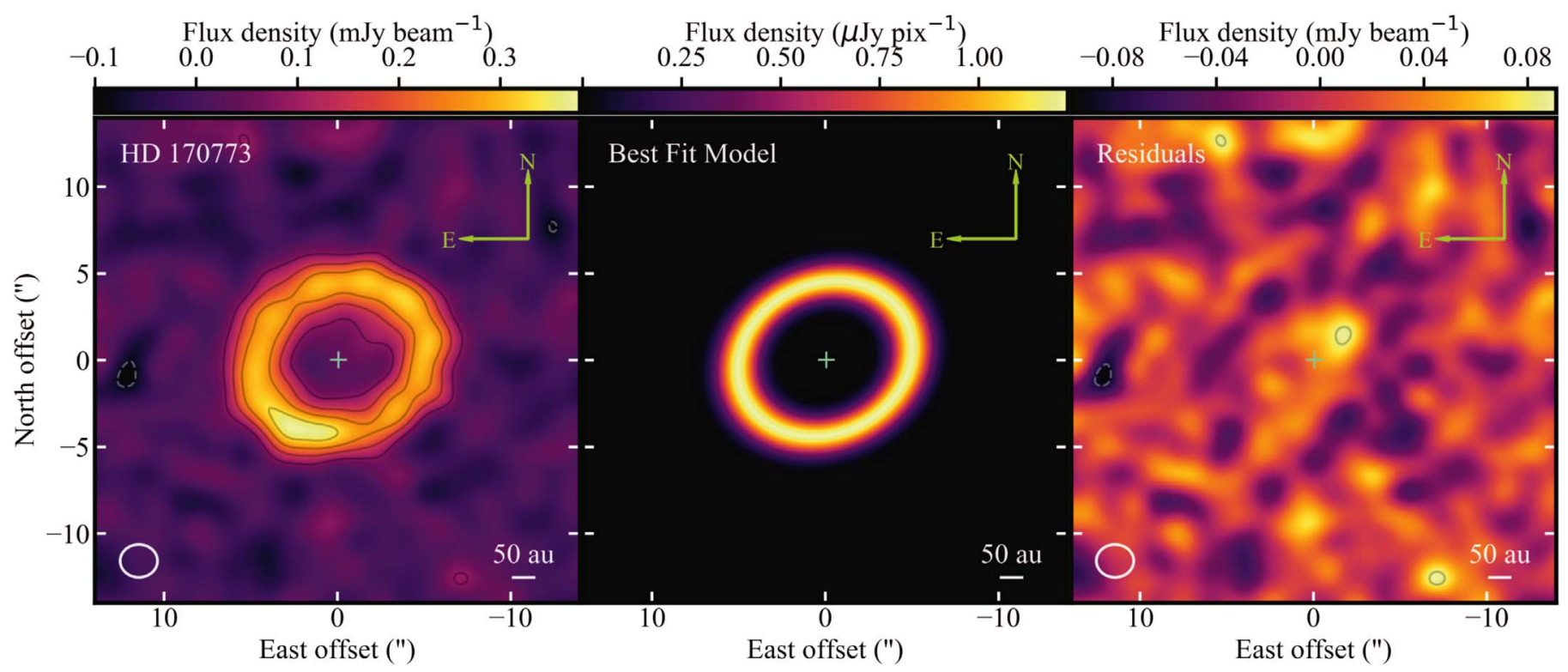

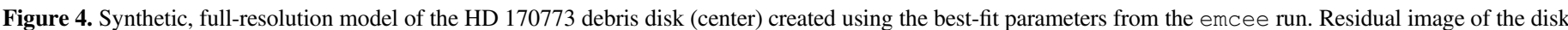

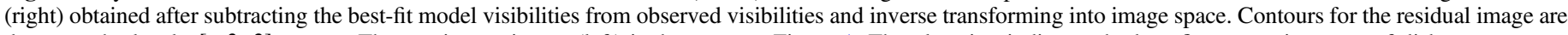
drawn at the levels: $[-3,3] \times \sigma_{\text {rms. }}$. The continuum image (left) is the same as Figure 1 . The plus sign indicates the best-fit geometric center of disk.
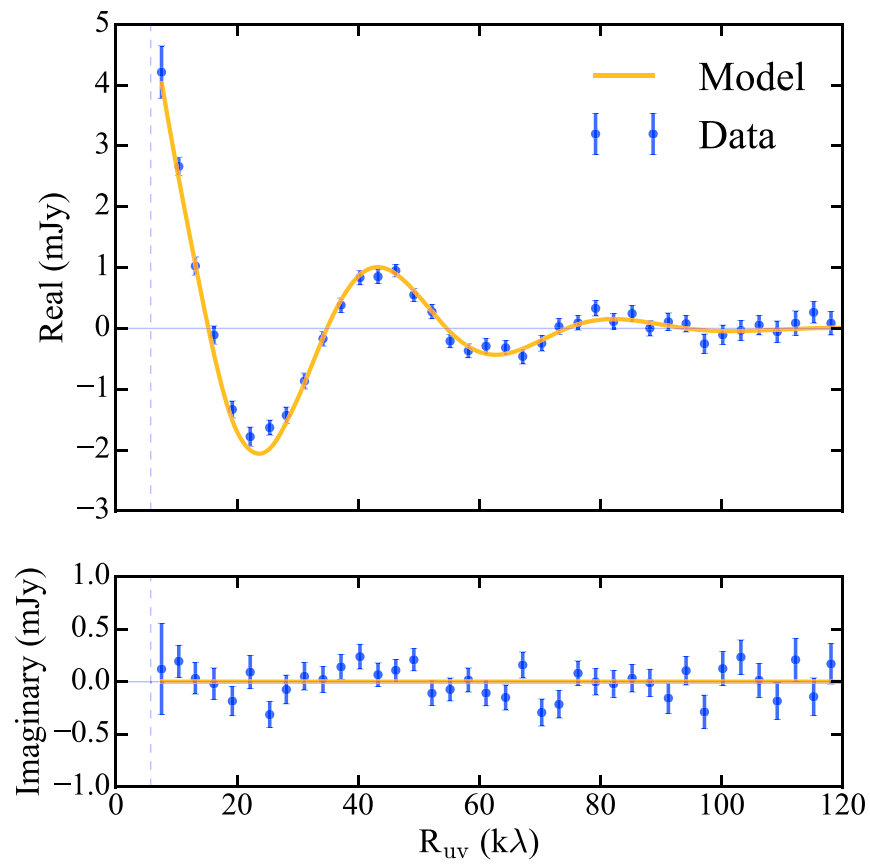

Figure 5. Real and imaginary part of the interferometric visibilities as a function of $u-v$ distance deprojected assuming the belt's best-fit position angle of $114^{\circ}$ and inclination of $33^{\circ}$. The best-fit model (orange line) is consistent with the data (blue error bars) within the uncertainties. The imaginary part of the visibilities being consistent with zero supports the lack of significant evidence for disk asymmetries.

young as $200 \mathrm{Myr}$ (Zuckerman \& Song 2004), then the total mass lost is $7.2 M_{\oplus}$.

Assuming a millimeter dust opacity of $\kappa_{\nu}=2.3 \mathrm{~cm}^{2} \mathrm{~g}^{-1}$ yields the mass in millimeter dust grains as $7.4 \times 10^{-1} M_{\oplus}$ (Equation (7) in Wyatt 2008). However, to produce the dust we see today, the size distribution must extend to much larger bodies, which will make the total belt mass much higher. For a steady-state size distribution described by a power law where $n(D) \propto D^{-3.5}$, the total mass of the collisional cascade can be
Table 4

Stellar Parameters and 68\% Uncertainties

\begin{tabular}{lcc}
\hline \hline Stellar Parameter & Derived Value & 68\% Confidence Interval \\
\hline Age $(\mathrm{Gyr})$ & 1.5 & $(+1.2,-0.7)$ \\
$\operatorname{Mass}\left(M_{\odot}\right)$ & 1.29 & $(+0.08,-0.08)$ \\
$\log (L)\left(L_{\odot}\right)$ & 0.558 & $(+0.006,-0.006)$ \\
$\mathrm{T}_{\text {eff }}(\mathrm{K})$ & 6551 & $(+32,-32)$ \\
$\operatorname{logg}(\mathrm{cgs})$ & 4.21 & $(+0.04,-0.04)$ \\
Radius $\left(R_{\odot}\right)$ & 1.477 & $(+0.022,-0.022)$ \\
\hline
\end{tabular}

linked to the size $D_{c} \mathrm{~km}$ of the largest bodies feeding the cascade (knowing the fractional luminosity, radius and minimum grain blowout size; see Equation (15) in Wyatt 2008). This leads to the expression $M_{\text {tot }}=75 \sqrt{D_{c}} M_{\oplus}$ for HD 170773. In addition, we can calculate the collisional timescale $t_{c}$ Myr of these largest bodies of size $D_{c}$ for a total solid mass within the cascade $M_{\text {tot }}$ (as a function of the known, or assumed, spatial properties of the disk, host-star mass, planetesimal strength, and mean planetesimal eccentricity; see Equation (16) in Wyatt 2008). Assuming a planetesimal strength of $150 \mathrm{~J} \mathrm{~kg}^{-1}$ and a mean planetesimal eccentricity of 0.05 yields $M_{\mathrm{tot}}=2.8 \times 10^{4} \times t_{c}^{-1} D_{c} M_{\oplus}$ for HD 170773 .

Combining the two equations for $M_{\text {tot }}$ leads to $D_{c}=$ $7 \times 10^{-6} \times t_{c}^{2} \mathrm{~km}$. As the largest bodies of size $D_{c}$ participating in the collisional cascade will be those whose timescale $t_{c}=t_{\text {age }}$ (assuming the collisional cascade has been ongoing for the age of the star), we let $t_{c}=1.5 \mathrm{Gyr}$ to find the size of the largest bodies needed to produce the dust we observe at the star's age. Here, that size is $D_{c}=16 \mathrm{~km}$, which we in turn use to find a total mass of $M_{\mathrm{tot}}=299 M_{\oplus}$. This estimate should be considered a lower limit on the total disk mass, as there could be larger planetesimals in the belt that are yet to suffer a collision and hence are not participating in the cascade. For an age range of $0.8-2.7 \mathrm{Gyr}$ (Table 4 ), the size of the largest bodies in the collisional cascade is $5-52 \mathrm{~km}$, which implies total disk masses of at least $175-539 M_{\oplus}$. 
To check whether such a large belt mass is reasonable, we compare it with the expectation from the Minimum Mass Solar Nebula (MMSN; Weidenschilling 1977; Hayashi 1981), linearly rescaled to account for a stellar host of mass $1.29 M_{\oplus}$ (as done in, e.g., Kenyon \& Bromley 2008). At 193 au around HD 170773, the expected MMSN-like surface density of solids would be $1.4 \times 10^{-2} \mathrm{~g} \mathrm{~cm}^{-2}$ (Equation (2.5) in Hayashi 1981), which leads to an estimated MMSN-like disk mass of $46 M_{\oplus}$ for a simple belt of large planetesimals of width 71 au and MMSN-like surface density. We therefore conclude that the large dust content and radius of the HD 170773 disk requires feeding from a planetesimal belt at least a few to an order of magnitude more massive than expected from a MMSN-like protoplanetary disk.

While keeping in mind the significant uncertainties in the inputs of the mass calculation (dominated by the system age, and by the unknown planetesimal strengths and eccentricities), the high masses obtained for the HD 170773 belt reinforce a disk mass problem recently highlighted for bright debris disks (Kennedy et al. 2018; Krivov et al. 2018). To reconcile observed belt masses with the expectation from the MMSN and observed protoplanetary disks, belts could either (1) have lower dynamical excitation than currently assumed (leading to lower eccentricities and higher dust masses at later ages); (2) have different strengths and size distributions than typically assumed; (3) have been collisionally evolving for a time shorter than the system age (requiring delayed stirring, e.g., Kenyon \& Bromley 2008); or (4) have sources of additional dust production beyond catastrophic collisions within the cascade considered here.

\subsection{Radius-Luminosity Relationship}

Matrà et al. (2018a) analyzed the 26 published debris disks resolved at millimeter wavelengths and found a significant correlation between their radii and host-star luminosities. We update the $R-L_{\star}$ relation by incorporating HD 170773 and any new studies of millimeter-resolved debris disks published since that time. The new additions are HD 32297 (MacGregor et al. 2018), HR 4796A (Kennedy et al. 2018), HD 92945 (Marino et al. 2019), TWA 7 (Bayo et al. 2018; Matrà et al. 2019a). The disks from the original sample with updated spatial properties are HD 107146 (Marino et al. 2018), HD 61005 (MacGregor et al. 2018), $\beta$ Pic (Matrà et al. 2019b), HD 131835 (Kral et al. 2018) and HR 8799 (Wilner et al. 2018). We find the updated correlation parameters to be $R_{1 L_{\odot}}=74 \pm 7 \mathrm{au}, \quad \alpha=0.16 \pm 0.05, \quad$ and $f_{\Delta R}=0.25_{-0.06}^{+0.08}$ (for more details on fitting the $R-L_{\star}$ relation, see Section 2 in Matrà et al. 2018a).

The HD 170773 debris disk is larger in radius than typical disks around late F/early $\mathrm{G}$ type stars located between 2 and 4 $L_{\odot}$ on the updated $R-L_{\star}$ relation plot (Figure 6). The disk instead lies closer to three other potentially outlying early $\mathrm{F}$ / late A disks, which are HR 8799 (Booth et al. 2016; Wilner et al. 2018), HD 95086 ( $\mathrm{Su}$ et al. 2017), and $\eta$ Crv (Marino et al. 2017). The significance of each of the four disks HD 170773, HR 8799, HD 95086 and $\eta$ Crv being outliers are $3.5 \sigma, 3.9 \sigma, 3.4 \sigma$, and $2.2 \sigma$, respectively. This was evaluated by comparing the best-fit disk radius to the probability distribution of radii at the corresponding host-star luminosity given the updated correlation parameters. While this indicates that these belts may truly form an outlying group, we caution that this conclusion is sensitive to the intrinsic scatter of radii about the

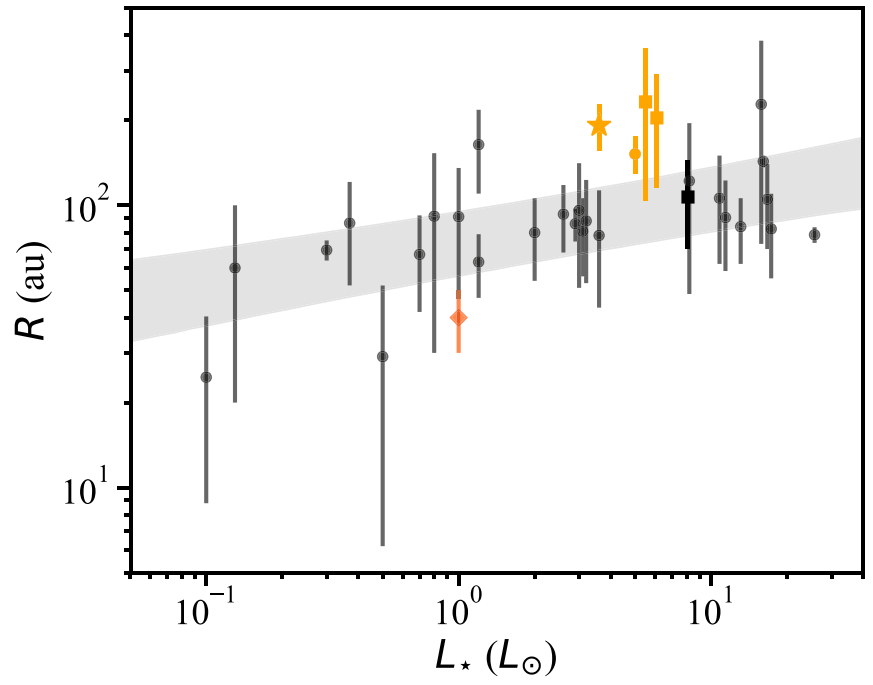

Figure 6. Radius-luminosity relation that expands upon the one published in Matrà et al. (2018a). The black points represent the published millimeterresolved belt radii and corresponding belt widths, compared with the host-star luminosity. The gray slope represents the effective $1 \sigma$ confidence interval for a range of power laws which describe the correlation. The four potential outliers are identified in orange, where HD 170773 is denoted with a star symbol. Systems that host directly imaged giant planets are denoted with a square. The red diamond represents our solar system's Kuiper Belt.

best-fit $R-L_{\star}$ model being modeled as a Gaussian (as opposed to, e.g., a top-hat) distribution (Section 2 of Matrà et al. 2018a).

An interesting property of this potentially outlying group is that half of the members, namely HR 8799 (Marois et al. 2008, 2010) and HD 95086 (Rameau et al. 2013), harbor giant planets that have been directly imaged. The two systems with detected giant planets have young age constraints $(\sim 40 \mathrm{Myr}$ for HR 8799 (Zuckerman et al. 2011) and 17 Myr for HD 95086 (Meshkat et al. 2013)) while the two with nondetections have older age constraints $(\sim 1.3$ Gyr for $\eta$ Crv (Mallik et al. 2003) and $\sim 1.5$ Gyr for HD 170773 (Section 3.5)), suggesting that the nondetections may be due to the giant planets having cooled and become too faint for direct detection (e.g., for $\eta \mathrm{Crv}$ see Lafrenière et al. 2007). By contrast, only two of the 27 $R-L_{\star}$ members not in this group host imaged planets (barring the solar system's Kuiper Belt for this consideration). These members are Fomalhaut (Kalas et al. 2008) and $\beta$ Pic (Lagrange et al. 2009; Dupuy et al. 2019), though the imaged companion of Fomalhaut is not likely in the giant planet mass regime (e.g., Kalas et al. 2013; Beust et al. 2014; Lawler et al. 2015). This tentative distinction could be alluding to some relationship between disk radii and long-period giant planet frequency, but more millimeter observations of disks and complementary direct imaging exoplanet surveys are needed to make a more robust approach to the problem.

\subsection{Exploring Hypothetical Exoplanetary System Architecture}

Stars that host both a debris disk and one or more exoplanets serve as critical test beds for studying the formation and evolution of planetary systems. Giant planets are present around at least $\sim 6 \%$ of stars with a detected debris disk compared with at least $\sim 0.7 \%$ of stars without a detected debris disk (Meshkat et al. 2017). To determine whether this distinction is due to observational bias or due to some intrinsic physical relation requires more observational efforts to detect disks and exoplanets for a larger sample of stars. 
A direct imaging survey carried out with Gemini Observatory (NICI Campaign) resulted in no detection of giant planets for HD 170773, indicating that any present companions were below the detection limits of $9.0 M_{\mathrm{Jup}}$ at 148 au and $13.4 M_{\mathrm{Jup}}$ at 74 au (Wahhaj et al. 2013, where hot-start models are used). The age used to calculate these hot-start mass upper limits was $200 \mathrm{Myr}$, which is less than the age we derive in this study and could thus mean that the mass upper limits may be higher. If an undetected planet around HD 170773 is both massive enough and orbiting at the necessary proximity to the inner edge of the disk to be clearing disk mass in its chaotic zone, then the inner radius of the disk, planet semimajor axis, and planet mass can be related by $R_{\mathrm{in}}=a_{\mathrm{pl}}+5 a_{\mathrm{pl}}\left(M_{\mathrm{pl}} / 3 M_{\star}\right)^{1 / 3} \quad($ Pearce \& Wyatt 2014, assuming a noneccentric orbit). Assuming this scenario and comparing against a linear interpolation of the published planet mass upper limits yields an estimate of $91 \mathrm{au}$ for the minimum planet semimajor axis. While a planet can approach an infinitesimally small mass at decreasingly short separations between it and the disk and still be consistent with these conditions, if we consider only the giant planet mass regime then a $1 M_{\text {Jup }}$ planet could plausibly be orbiting at $120 \mathrm{au}$. Future deeper imaging with higher contrast sensitivity will be needed to reveal the presence of such an orbiting companion.

\section{Conclusion}

We used ALMA to obtain the first millimeter-resolved observations of the dust and gas around HD 170773 as part of the REASONS survey. We forward-modeled the disk as an axisymmetric thin Gaussian ring and found the disk width to be $71_{-4}^{+4}$ au and the radius from the host star to be $193_{-3}^{+2}$ au. The spatial properties are consistent with previous studies of this disk, and reveal that HD 170773 hosts a large and narrow debris disk when compared with the currently known disk population.

We also searched for any $\mathrm{CO}$ and $\mathrm{CN}$ gas released by exocomets in the system. We set upper limits on their gas mass, which allow us to constrain the mass fraction of $\mathrm{CO}$ and $\mathrm{HCN}$ ice in exocomets to $<77 \%$ and $<3 \%$, respectively. These upper limits still allow for HD 170773 to be hosting icy exocomets with compositions analogous to the solar system and other known gas-bearing exocometary belts.

The disk characteristics were used together with constraints on the stellar parameters from Gaia DR2 to estimate some of the system properties. We found that bodies must be at least $16 \mathrm{~km}$ in diameter to sustain a steady-state collisional cascade producing the currently observed dust levels after $1.5 \mathrm{Gyr}$ of evolution. This $16 \mathrm{~km}$ size would lead to a total mass in the collisional cascade of $\sim 300 M_{\oplus}$. This is almost an order of magnitude larger than expected from a MMSN-like protoplanetary disk, which (barring significant uncertainties in some of the assumed parameters) provides further support for the presence of a disk mass problem for bright debris disks (Kennedy et al. 2018; Krivov et al. 2018).

In the context of the $R-L_{\star}$ relation of planetesimal belts from mm-wave imaging, HD 170773 is part of a group of potentially outlying large F star disks. Interestingly, two of the four potential outliers in this group also host directly imaged long-period giant planets (versus $1 / 27$ in the remaining belt population), which may suggest a relationship between the frequency of long-period giant planets and the presence of large debris disks. Around HD 170773, we find that a hypothetical long-period giant planet clearing material inward of the disk's inner edge should lie beyond 91 au to remain below current direct imaging detection limits.

REASONS and other future surveys will play a vital role in characterizing the spatial properties of debris disks. These surveys should be complemented by direct imaging surveys to find systems which host both exoplanets and a debris disk, providing an invaluable laboratory to further analyze the dynamics of exoplanetary systems.

A.G.S. acknowledges support from the SAO REU program, funded in part by the National Science Foundation REU and Department of Defense ASSURE programs under NSF grant No. AST-1659473, and by the Smithsonian Institution. L.M. acknowledges support from the Smithsonian Institution as a Submillimeter Array (SMA) Fellow. G.M.K. is supported by the Royal Society as a Royal Society University Research Fellow. Cd.B. acknowledges the funding of his sabbatical position through the Mexican national council for science and technology (CONACYT grant CVU No.448248). M.B. acknowledges support from the Deutsche Forschungsgemeinschaft through project Kr 2164/15-1. J.M.C. acknowledges support from the National Aeronautics and Space Administration under grant No. 15XRP15_20140 issued through the Exoplanets Research Program. C.L.D. acknowledges support from the ERC Starting Grant "ImagePlanetFormDiscs" (grant agreement No. 639889). J.P.M. acknowledges research support by the Ministry of Science and Technology of Taiwan under grants MOST104-2628-M001-004-MY3 and MOST107-2119-M-001-031-MY3, and Academia Sinica under grant AS-IA-106-M03.

This paper makes use of the following ALMA data: JAO. ALMA\#2018.1.00200.S. ALMA is a partnership of ESO (representing its member states), NSF (USA) and NINS (Japan), together with NRC (Canada), MOST and ASIAA (Taiwan), and KASI (Republic of Korea), in cooperation with the Republic of Chile. The Joint ALMA Observatory is operated by ESO, AUI/NRAO and NAOJ. The National Radio Astronomy Observatory is a facility of the National Science Foundation operated under cooperative agreement by Associated Universities, Inc.

This work has made use of data from the European Space Agency (ESA) mission Gaia (https://www.cosmos.esa.int/ gaia), processed by the Gaia Data Processing and Analysis Consortium (DPAC, https://www.cosmos.esa.int/web/gaia/ dpac/consortium). Funding for the DPAC has been provided by national institutions, in particular the institutions participating in the Gaia Multilateral Agreement.

This research has made use of the NASA Exoplanet Archive, which is operated by the California Institute of Technology, under contract with the National Aeronautics and Space Administration under the Exoplanet Exploration Program.

This research has made use of the SIMBAD database, operated at CDS, Strasbourg, France.

This research has made use of NASA's Astrophysics Data System Bibliographic Services.

Facility: ALMA.

Software: CASA (McMullin et al. 2007), galario (Tazzari et al. 2018), emcee (Foreman-Mackey et al. 2013), matplotlib (Hunter 2007), corner (Foreman-Mackey 2016), astropy (Astropy Collaboration et al. 2013, 2018), numpy 
(van der Walt et al. 2011), scipy (Oliphant 2007), multinest (Feroz et al. 2009).

\section{ORCID iDs}

Aldo G. Sepulveda (1) https://orcid.org/0000-0002-8621-2682 Luca Matrà (i) https://orcid.org/0000-0003-4705-3188

Grant M. Kennedy (10 https://orcid.org/0000-0001-6831-7547

Karin I. Öberg (1) https://orcid.org/0000-0001-8798-1347

David J. Wilner (1) https://orcid.org/0000-0003-1526-7587

Sebastián Marino (1) https://orcid.org/0000-0002-5352-2924

John M. Carpenter (i) https://orcid.org/0000-0003-2251-0602

Claire L. Davies (i) https://orcid.org/0000-0001-9764-2357

Steve Ertel (1) https://orcid.org/0000-0002-2314-7289

Jonathan P. Marshall (i) https://orcid.org/0000-0001-

6208-1801

Julien Milli (ib https://orcid.org/0000-0001-9325-2511

Mark C. Wyatt (i) https://orcid.org/0000-0001-9064-5598

Meredith A. MacGregor (i) https://orcid.org/0000-0001-

7891-8143

Brenda C. Matthews (i) https://orcid.org/0000-0003-

3017-9577

\section{References}

Astropy Collaboration, Price-Whelan, A. M., Sipőcz, B. M., et al. 2018, AJ, 156,123

Astropy Collaboration, Robitaille, T. P., Tollerud, E. J., et al. 2013, A\&A, 558, A33

Augereau, J. C., Nelson, R. P., Lagrange, A. M., Papaloizou, J. C. B., \& Mouillet, D. 2001, A\&A, 370, 447

Backman, D. E., \& Paresce, F. 1993, in Protostars and Planets III, ed. E. H. Levy \& J. I. Lunine (Tucson, AZ: Univ. Arizona Press), 1253

Bailer-Jones, C. A. L., Rybizki, J., Fouesneau, M., Mantelet, G., \& Andrae, R. 2018, AJ, 156, 58

Ballering, N. P., Rieke, G. H., Su, K. Y. L., \& Gáspár, A. 2017, ApJ, 845, 120

Bayo, A., Olofsson, J., Matra, L., et al. 2019, MNRAS, 486, 5552

Beust, H., Augereau, J.-C., Bonsor, A., et al. 2014, A\&A, 561, A43

Booth, M., Dent, W. R. F., Jordán, A., et al. 2017, MNRAS, 469, 3200

Booth, M., Jordán, A., Casassus, S., et al. 2016, MNRAS, 460, L10

Bowler, B. P. 2016, PASP, 128, 102001

Bressan, A., Marigo, P., Girardi, L., et al. 2012, MNRAS, 427, 127

Chen, C. H., Mittal, T., Kuchner, M., et al. 2014a, ApJS, 211, 25

Chen, Y., Bressan, A., Girardi, L., et al. 2015, MNRAS, 452, 1068

Chen, Y., Girardi, L., Bressan, A., et al. 2014b, MNRAS, 444, 2525

Cutri, R. M., Skrutskie, M. F., van Dyk, S., et al. 2003, 2MASS All Sky Catalog of Point Sources (Washington, DC: NASA)

del Burgo, C., \& Allende Prieto, C. 2016, MNRAS, 463, 1400

del Burgo, C., \& Allende Prieto, C. 2018, MNRAS, 479, 1953

Dupuy, T. J., Brandt, T. D., Kratter, K. M., \& Bowler, B. P. 2019, ApJL, 871, L4

ESA 1997, The HIPPARCOS and TYCHO Catalogues. Astrometric and Photometric Star Catalogues Derived from the ESA HIPPARCOS Space Astrometry Mission, Vol. 1200 (Noordwijk: ESA)

Evans, D. W., Riello, M., De Angeli, F., et al. 2018, A\&A, 616, A4

Feroz, F., Hobson, M. P., \& Bridges, M. 2009, MNRAS, 398, 1601

Foreman-Mackey, D. 2016, JOSS, 1, 24

Foreman-Mackey, D., Hogg, D. W., Lang, D., \& Goodman, J. 2013, PASP, 125,306

Gaia Collaboration, Brown, A. G. A., Vallenari, A., et al. 2016, A\&A, 595, A2 Gaia Collaboration, Brown, A. G. A., Vallenari, A., et al. 2018, A\&A, 616, A1 Goodman, J., \& Weare, J. 2010, Commun. Appl. Math. Comput. Sci., 5, 65 Gray, R. O., Corbally, C. J., Garrison, R. F., et al. 2006, AJ, 132, 161 Hayashi, C. 1981, PThPS, 70, 35

Heays, A. N., Bosman, A. D., \& van Dishoeck, E. F. 2017, A\&A, 602, A105 Høg, E., Fabricius, C., Makarov, V. V., et al. 2000, A\&A, 355, L27

Holland, W. S., Matthews, B. C., Kennedy, G. M., et al. 2017, MNRAS, 470,3606
Hughes, A. M., Duchêne, G., \& Matthews, B. C. 2018, ARA\&A, 56, 541 Hunter, J. D. 2007, CSE, 9, 90

Husser, T.-O., Wende-von Berg, S., Dreizler, S., et al. 2013, A\&A, 553, A6 Ishihara, D., Onaka, T., Kataza, H., et al. 2010, A\&A, 514, A1

Kalas, P., Graham, J. R., Chiang, E., et al. 2008, Sci, 322, 1345

Kalas, P., Graham, J. R., \& Clampin, M. 2005, Natur, 435, 1067

Kalas, P., Graham, J. R., Fitzgerald, M. P., \& Clampin, M. 2013, ApJ, 775, 56

Kennedy, G. M., Marino, S., Matrà, L., et al. 2018, MNRAS, 475, 4924

Kennedy, G. M., \& Wyatt, M. C. 2014, MNRAS, 444, 3164

Kenyon, S. J., \& Bromley, B. C. 2008, ApJS, 179, 451

Kral, Q., Marino, S., Wyatt, M. C., Kama, M., \& Matra, L. 2018, arXiv:1811. 08439

Krivov, A. V., Ide, A., Löhne, T., Johansen, A., \& Blum, J. 2018, MNRAS, 474,2564

Lafrenière, D., Doyon, R., Marois, C., et al. 2007, ApJ, 670, 1367

Lagrange, A.-M., Gratadour, D., Chauvin, G., et al. 2009, A\&A, 493, L21

Lawler, S. M., Greenstreet, S., \& Gladman, B. 2015, ApJL, 802, L20

Lebouteiller, V., Barry, D. J., Spoon, H. W. W., et al. 2011, ApJS, 196, 8

Lindegren, L., Lammers, U., Bastian, U., et al. 2016, A\&A, 595, A4

MacGregor, M. A., Matrà, L., Kalas, P., et al. 2017, ApJ, 842, 8

MacGregor, M. A., Weinberger, A. J., Hughes, A. M., et al. 2018, ApJ, 869, 75

MacGregor, M. A., Wilner, D. J., Andrews, S. M., Lestrade, J.-F., \& Maddison, S. 2015, ApJ, 809, 47

MacGregor, M. A., Wilner, D. J., Chandler, C., et al. 2016, ApJ, 823, 79

Mallik, S. V., Parthasarathy, M., \& Pati, A. K. 2003, A\&A, 409, 251

Marino, S., Carpenter, J., Wyatt, M. C., et al. 2018, MNRAS, 479, 5423

Marino, S., Matrà, L., Stark, C., et al. 2016, MNRAS, 460, 2933

Marino, S., Wyatt, M. C., Panić, O., et al. 2017, MNRAS, 465, 2595

Marino, S., Yelverton, B., Booth, M., et al. 2019, MNRAS, 484, 1257

Marois, C., Macintosh, B., Barman, T., et al. 2008, Sci, 322, 1348

Marois, C., Zuckerman, B., Konopacky, Q. M., Macintosh, B., \& Barman, T. 2010, Natur, 468, 1080

Marshall, J. P., Milli, J., Choquet, É., et al. 2018, ApJ, 869, 10

Matrà, L., MacGregor, M. A., Kalas, P., et al. 2017, ApJ, 842, 9

Matrà, L., Marino, S., Kennedy, G. M., et al. 2018a, ApJ, 859, 72

Matrà, L., Öberg, K. I., Wilner, D. J., Olofsson, J., \& Bayo, A. 2019a, AJ, 157,117

Matrà, L., Panić, O., Wyatt, M. C., \& Dent, W. R. F. 2015, MNRAS, 447, 3936

Matrà, L., Wilner, D. J., Öberg, K. I., et al. 2018b, ApJ, 853, 147

Matrà, L., Wyatt, M. C., Wilner, D. J., et al. 2019b, AJ, 157, 135

McMullin, J. P., Waters, B., Schiebel, D., Young, W., \& Golap, K. 2007, in ASP Conf. Ser. 376, Astronomical Data Analysis Software and Systems XVI, ed. R. A. Shaw, F. Hill, \& D. J. Bell (San Francisco, CA: ASP), 127 Mermilliod, J. C. 2006, yCat, 2168

Meshkat, T., Bailey, V., Rameau, J., et al. 2013, ApJL, 775, L40

Meshkat, T., Mawet, D., Bryan, M. L., et al. 2017, AJ, 154, 245

Montesinos, B., Eiroa, C., Krivov, A. V., et al. 2016, A\&A, 593, A51

Moór, A., Kóspál, Á., Ábrahám, P., et al. 2015, MNRAS, 447, 577

Mumma, M. J., \& Charnley, S. B. 2011, ARA\&A, 49, 471

Nilsson, R., Liseau, R., Brandeker, A., et al. 2010, A\&A, 518, A40

Oliphant, T. E. 2007, CSE, 9, 10

Paunzen, E. 2015, A\&A, 580, A23

Pearce, T. D., \& Wyatt, M. C. 2014, MNRAS, 443, 2541

Rameau, J., Chauvin, G., Lagrange, A.-M., et al. 2013, ApJL, 776, L17

Rotundi, A., Sierks, H., Della Corte, V., et al. 2015, Sci, 347, aaa3905

Sadakane, K., \& Nishida, M. 1986, PASP, 98, 685

Schulz, B., Marton, G., Valtchanov, I., et al. 2017, arXiv:1706.00448

Schwarz, G. 1978, AnSta, 6, 461

Sibthorpe, B., Kennedy, G. M., Wyatt, M. C., et al. 2018, MNRAS, 475, 3046

Su, K. Y. L., MacGregor, M. A., Booth, M., et al. 2017, AJ, 154, 225

Tang, J., Bressan, A., Rosenfield, P., et al. 2014, MNRAS, 445, 4287

Tazzari, M., Beaujean, F., \& Testi, L. 2018, MNRAS, 476, 4527

van der Walt, S., Colbert, S. C., \& Varoquaux, G. 2011, CSE, 13, 22

Wahhaj, Z., Liu, M. C., Nielsen, E. L., et al. 2013, ApJ, 773, 179

Weidenschilling, S. J. 1977, Ap\&SS, 51, 153

Wilner, D. J., MacGregor, M. A., Andrews, S. M., et al. 2018, ApJ, 855, 56

Wright, E. L., Eisenhardt, P. R. M., Mainzer, A. K., et al. 2010, AJ, 140, 1868 Wyatt, M. C. 2008, ARA\&A, 46, 339

Wyatt, M. C. 2018, Debris Disks: Probing Planet Formation (Cham: Springer) Wyatt, M. C., Clarke, C. J., \& Booth, M. 2011, CeMDA, 111, 1

Zuckerman, B., Rhee, J. H., Song, I., \& Bessell, M. S. 2011, ApJ, 732, 61

Zuckerman, B., \& Song, I. 2004, ApJ, 603, 738 